\title{
Thermodynamics at work: The pressure derivative of the specific heat
}

\section{J. Güémez}

Departamento de Física Aplicada, Universidad de Cantabria, E-39005 Santander, Spain

\section{Fiolhais and M. Fiolhais}

Departamento de Física and Centro de Física Computacional, Universidade de Coimbra, P-3000 Coimbra, Portugal

(Received 25 February 1999; accepted 2 July 1999)

\begin{abstract}
Thermodynamics relates measurable quantities such as thermal coefficients and specific heats. The first law, which implies that the enthalpy is a function of state, yields a relation for the pressure derivative of the specific heat $c_{P}$. The second law gives a simpler and well-known relation for this pressure derivative. We compare the values of the pressure derivative of $c_{P}$ obtained from the first and second laws to the values obtained from measurements for water at different pressures. The comparison illustrates the scope and methodology of thermodynamics. (C) 1999 American Association of Physics Teachers.
\end{abstract}

\section{INTRODUCTION}

Thermodynamics is based on a small number of principles and provides a formalism which relates the various thermal coefficients and specific heats for any substance in equilibrium. ${ }^{1}$ Examples of typical thermodynamic relations are the reciprocity theorem, arising from the zeroth law, Reech's relation, arising from the first law, and Mayer's relation, arising from the second law. These relations can be summarized as follows.

From the zeroth law, the reciprocity theorem for a PVT (pressure, volume, and temperature) system states that

$$
\beta=\frac{\alpha}{\kappa_{T} P} \quad \text { (reciprocity theorem), }
$$

where $\beta \equiv(1 / P)(\partial P / \partial T)_{V}$ is the relative pressure coefficient, $\alpha \equiv(1 / \mathrm{v})(\partial \mathrm{v} / \partial T)_{P}$ is the (cubic) thermal expansion coefficient, $\mathrm{V}$ is the specific volume, and $\kappa_{T} \equiv-(1 / \mathrm{v})$ $\times(\partial \mathrm{v} / \partial P)_{T}$ is the isothermal compressibility. Equation (1) follows readily from the existence of a thermal equation of state, because

$$
\left(\frac{\partial \mathrm{v}}{\partial P}\right)_{T}\left(\frac{\partial P}{\partial T}\right)_{V}\left(\frac{\partial T}{\partial \mathrm{V}}\right)_{P}=-1 .
$$

It is interesting to show the validity of Eq. (1) using only experimental data. A pedagogical example using a rubber strip (a tension, length, and temperature system) is discussed in Ref. 2. If Eq. (2) were not experimentally fulfilled, the system would not be in equilibrium. ${ }^{3}$ Equation (1) can be used to obtain a coefficient such as $\beta$, which is difficult to measure, from $\alpha$ and $\kappa_{T}$, which are easier to measure. (It is difficult in practice to keep the volume constant when the temperature is being changed by heating.)

We can derive Reech's relation from the first law, ${ }^{4}$ which can be expressed as $d u=\delta q-P d \mathrm{v}$, where $u$ is the specific internal energy and $q$ is the (specific) heat transfer. Reech's relation $^{5}$ states that

$$
c_{V}=c_{P} \frac{\kappa_{s}}{\kappa_{T}} \quad \text { (Reech's relation), }
$$

where $\quad c_{V} \equiv(\delta q / \partial T)_{V}=(\partial u / \partial T)_{V} \quad$ and $\quad c_{P} \equiv(\delta q / \partial T)_{P}$ $=(\partial h / \partial T)_{P}$ are the specific heats at constant volume and pressure, respectively, the (specific) enthalpy $h=u+P \vee$, and $\kappa_{s} \equiv-(1 / \mathrm{v})(\partial \mathrm{v} / \partial P)_{s}$ is the adiabatic compressibility. ${ }^{6}$ Equation (3) implies that the ratio of the specific heats at constant pressure and at constant volume ${ }^{7,8}$ equals the ratio of the isothermal and adiabatic compressibilities. ${ }^{9}$ If these quantities are measured independently, we could verify the first law. Equation (3) can be used to determine the specific heat, $c_{V}$, which is difficult to measure because of the difficulty of keeping the volume constant.

Mayer's relation follows from the second and first laws and can be expressed as: ${ }^{10}$

$$
c_{V}=c_{P}-\frac{T \alpha^{2} \mathrm{v}}{\kappa_{T}} \quad \text { (Mayer's relation). }
$$

Equation (4) is one of the most important relations of thermodynamics. ${ }^{11}$ The independent measurement of the coefficients appearing in Eq. (4) allows us to verify the first and second laws of thermodynamics. We may also use Eq. (4), instead of Eq. (3) to obtain $c_{V}$.

Equations (1), (3), and (4) imply that among the thermal coefficients $\alpha, \kappa_{T}, \kappa_{s}$, and $\beta$, and the specific heats $c_{P}$ and $c_{V}$, only three can be considered independent. For example, if we choose $\kappa_{T}, \alpha$, and $c_{P}$, then $c_{V}$ could be obtained from Eq. (4) and $\beta$ and $\kappa_{s}$ can be obtained from Eqs. (1) and (3).

It is difficult to verify any of the relations, Eqs. (1), (3), or (4) directly, because at least one of the quantities in each relation is difficult to measure. However, we can test experimental data for thermodynamic consistency by considering equations for $\left(\partial c_{P} / \partial P\right)_{T}$. In this paper, we show how to obtain $\left(\partial c_{P} / \partial P\right)_{T}$ using only the first law. We will arrive at the relation

$$
\left(\frac{\partial c_{P}}{\partial P}\right)_{T}=\alpha \mathrm{v}-\left[\left(c_{P}-c_{V}\right) \frac{\kappa_{T}}{\alpha}\right]^{\prime},
$$

where the prime denotes differentiation with respect to temperature at constant pressure. If we substitute Eq. (4) in Eq. (5), we obtain another relation

$$
\left(\frac{\partial c_{P}}{\partial P}\right)_{T}=-T \vee\left(\alpha^{\prime}+\alpha^{2}\right) .
$$

Equation (6) is well-known, but Eq. (5) is not. Note that the latter relation provides an experimental check of the first law. We emphasize that the first law can be verified independently of the second. Thus, we may imagine a hypothetical reversible world where the first law holds but not the second. 
Table I. Experimental data for water at $P_{0}=101.3 \mathrm{kPa}$ and temperatures ranging from the ice point to the boiling point (Ref. 15 ). The first six columns are direct experimental data and the last three columns are determined indirectly. The specific heat in column 8 was obtained from the relation $c_{V}=c_{P} \kappa_{S} / \kappa_{T}$ and the specific heat in column 9 was calculated by using $c_{V}=c_{P}-T \alpha^{2} \mathrm{~V} / k_{T}$.

\begin{tabular}{|c|c|c|c|c|c|c|c|c|}
\hline $\begin{array}{c}T \\
{ }^{\circ} \mathrm{C}\end{array}$ & $\begin{array}{l}\mathrm{v} \times 10^{3} \\
\mathrm{~m}^{3} \mathrm{~kg}^{-1}\end{array}$ & $\begin{array}{c}\alpha \times 10^{6} \\
\mathrm{~K}^{-1}\end{array}$ & $\begin{array}{c}\kappa_{T} \times 10^{10} \\
\mathrm{~Pa}^{-1}\end{array}$ & $\begin{array}{c}\kappa_{S} \times 10^{10} \\
\mathrm{~Pa}^{-1}\end{array}$ & $\begin{array}{c}c_{P} \\
\mathrm{~J} \mathrm{~kg}^{-1} \mathrm{~K}^{-1}\end{array}$ & $\begin{array}{c}\beta[\text { Eq. (1) }] \\
\mathrm{K}^{-1}\end{array}$ & $\begin{array}{c}c_{V}[\mathrm{Eq} .(3)] \\
\mathrm{J} \mathrm{kg}^{-1} \mathrm{~K}^{-1}\end{array}$ & $\begin{array}{c}c_{V}[\mathrm{Eq} .(4)] \\
\mathrm{J} \mathrm{kg}^{-1} \mathrm{~K}^{-1}\end{array}$ \\
\hline 0 & 1.00016 & -67.89 & 5.0885 & 5.0855 & 4217.6 & -1.3171 & 4215.1 & 4215.1 \\
\hline 10 & 1.00030 & 87.96 & 4.7810 & 4.7758 & 4192.1 & 1.8162 & 4187.5 & 4187.5 \\
\hline 20 & 1.00179 & 206.80 & 4.5891 & 4.5591 & 4181.8 & 4.4485 & 4154.5 & 4154.5 \\
\hline 30 & 1.00437 & 303.23 & 4.4770 & 4.4100 & 4178.4 & 6.6861 & 4115.9 & 4115.9 \\
\hline 40 & 1.00784 & 385.30 & 4.4240 & 4.3119 & 4178.5 & 8.5975 & 4072.6 & 4072.6 \\
\hline 50 & 1.01211 & 457.60 & 4.4174 & 4.2536 & 4180.6 & 10.226 & 4025.6 & 4025.6 \\
\hline 60 & 1.01709 & 523.07 & 4.4496 & 4.2281 & 4184.3 & 11.605 & 3976.0 & 3976.0 \\
\hline 70 & 1.02272 & 583.74 & 4.5161 & 4.2307 & 4189.5 & 12.760 & 3924.7 & 3924.7 \\
\hline 80 & 1.02900 & 641.11 & 4.6143 & 4.2584 & 4196.3 & 13.716 & 3872.6 & 3872.6 \\
\hline 90 & 1.03589 & 696.24 & 4.7430 & 4.3093 & 4205.0 & 14.491 & 3820.5 & 3820.5 \\
\hline 100 & 1.04341 & 750.30 & 4.9018 & 4.3819 & 4215.9 & 15.110 & 3768.7 & 3768.8 \\
\hline
\end{tabular}

In Sec. II we derive these two results, and in Sec. III we compare them using experimental data for liquid water. Some conclusions and suggested problems are given in Sec. IV.

\section{THERMODYNAMIC RELATIONS}

The first law asserts that measurements of the variations of the internal energy in an isolated thermal system can be reduced to a pure mechanical problem. ${ }^{12}$ This statement assumes the existence of adiabatic walls. Furthermore, these variations are independent of the process from the initial to the final state, and thus the internal energy is a function of state.

In practice, the most convenient independent variables are the intensive quantities $T$ and $P .{ }^{13}$ For this reason, the enthalpy should be used instead of internal energy. From the first law, we find the following expression relating the derivatives of the enthalpy:

$$
\left(\frac{\partial h}{\partial T}\right)_{\mathrm{V}}=c_{V}+\mathrm{v}\left(\frac{\partial P}{\partial T}\right)_{\mathrm{V}}=\left(\frac{\partial h}{\partial T}\right)_{P}+\left(\frac{\partial h}{\partial P}\right)_{T}\left(\frac{\partial P}{\partial T}\right)_{\mathrm{V}} .
$$

Using the definitions of $c_{P}$ and $\beta$ and Eq. (1), we obtain

$$
c_{P}-c_{V}=\frac{\alpha}{\kappa_{T}}\left[\mathrm{v}-\left(\frac{\partial h}{\partial P}\right)_{T}\right],
$$

which may be written as:

$$
\left(\frac{\partial h}{\partial P}\right)_{T}=\mathrm{v}-\left(c_{P}-c_{V}\right) \frac{\kappa_{T}}{\alpha} .
$$

Taking the derivative of this expression with respect to the temperature and applying Schwartz's theorem (the value of mixed derivatives is independent of the order in which the derivatives are taken) to the enthalpy leads directly to

$$
\frac{\partial^{2} h}{\partial T \partial P}=\alpha \mathrm{v}-\left[\left(c_{P}-c_{V}\right) \frac{\kappa_{T}}{\alpha}\right]^{\prime}=\frac{\partial^{2} h}{\partial P \partial T}=\left(\frac{\partial c_{P}}{\partial P}\right)_{T},
$$

which is the same as Eq. (5). This result was given in a different form by Max Planck in his classical monograph. ${ }^{14}$ In Appendix A, we derive Planck's result and show its equivalence to Eq. (5).

Although Eq. (6) can be obtained by substituting Eq. (4) into Eq. (5), another way of deriving Eq. (6) is the following. If we combine the second and the first laws, we have

$$
d s=\frac{1}{T} d h-\frac{\mathrm{v}}{T} d P=\frac{1}{T}\left[\left(\frac{\partial h}{\partial P}\right)_{T}-\mathrm{v}\right] d P+\frac{c_{P}}{T} d T .
$$

Applying Schwartz's theorem to the entropy, we obtain

$$
\frac{1}{T}\left(\frac{\partial c_{P}}{\partial P}\right)_{T}=-\frac{1}{T^{2}}\left[\left(\frac{\partial h}{\partial P}\right)_{T}-\mathrm{v}\right]+\frac{1}{T} \frac{\partial^{2} h}{\partial T \partial P}-\frac{1}{T}\left(\frac{\partial \mathrm{v}}{\partial T}\right)_{P} .
$$

Using Eq. (10), we obtain

$$
\left(\frac{\partial h}{\partial P}\right)_{T}=\mathrm{v}-T\left(\frac{\partial \mathrm{v}}{\partial T}\right)_{P}=\mathrm{v}(1-T \alpha) .
$$

This important result may be called the compatibility condition because it equates two different kinds of quantities: a derivative of the enthalpy on the left-hand side and a function obtained directly from the equation of state on the righthand side. Equation (6) is obtained by taking the temperature derivative of Eq. (13) at constant pressure.

\section{ANALYSIS OF EXPERIMENTAL DATA}

In the following we obtain the derivative $\left(\partial c_{P} / \partial P\right)_{T}$ for water using data for the thermal coefficients and specific heats and various thermodynamic relations. We have chosen water because accurate data are available for a wide range of temperatures and pressures. The data in Table I are for liquid water at $101.3 \mathrm{kPa}$ (normal atmospheric pressure). ${ }^{15}$ The data in Table II is for liquid water in the pressure range 50 $\times 10^{5} \mathrm{~Pa}$ to $200 \times 10^{5} \mathrm{~Pa}^{16}$

The specific volume, $\mathrm{v}$, and the thermal expansion coefficient, $\alpha$, given in Table I can be measured directly. To obtain $\kappa_{T}$, specific volumes at different pressures are needed (see Table II). The coefficient $\kappa_{s}$ is measured from the speed of sound. The enthalpy, $h$, and the specific heat, $c_{P}$, are measured using an electric calorimeter.

On the other hand, $\beta$ and $c_{V}$ are difficult to measure directly, because the volume of water changes upon heating. It is convenient, therefore, to use, respectively, the reciprocity theorem, Eq. (1) and Eq. (3) to obtain them. Table I shows the resulting values for $\beta$ and $c_{V}$. In the last column of Table I we show the values of $c_{V}$ as calculated from Eq. (4). The values of $c_{V}$ turn out to be the same (within 5 digits) using either relation. 
Table II. Experimental specific volumes, $\mathrm{v} \times 10^{3}$ (in $\mathrm{m}^{3} \mathrm{~kg}^{-1}$ ), and enthalpies, $h \times 10^{-3}$ (in J kg${ }^{-1}$ ), for water at different pressures and temperatures (in $\left.{ }^{\circ} \mathrm{C}\right)$ (Ref. 16).

\begin{tabular}{|c|c|c|c|c|c|c|c|c|}
\hline \multirow[b]{2}{*}{$T$} & \multicolumn{2}{|c|}{$P=5 \mathrm{MPa}$} & \multicolumn{2}{|c|}{$P=10 \mathrm{MPa}$} & \multicolumn{2}{|c|}{$P=15 \mathrm{MPa}$} & \multicolumn{2}{|c|}{$P=20 \mathrm{MPa}$} \\
\hline & v & $h$ & v & $h$ & v & $h$ & v & $h$ \\
\hline 0 & 0.9977 & 5.03 & 0.9952 & 10.04 & 0.9928 & 15.04 & 0.9904 & 20.00 \\
\hline 20 & 0.9995 & 88.65 & 0.9972 & 93.33 & 0.9950 & 97.99 & 0.9928 & 102.58 \\
\hline 40 & 1.0056 & 171.98 & 1.0034 & 176.38 & 1.0013 & 180.78 & 0.9992 & 185.15 \\
\hline 60 & 1.0149 & 255.30 & 1.0127 & 259.49 & 1.0105 & 263.67 & 1.0084 & 267.85 \\
\hline 80 & 1.0268 & 338.85 & 1.0245 & 342.84 & 1.0222 & 346.81 & 1.0199 & 350.80 \\
\hline 100 & 1.0410 & 422.81 & 1.0385 & 426.51 & 1.0361 & 430.28 & 1.0337 & 434.06 \\
\hline
\end{tabular}

To obtain $\left(\partial c_{P} / \partial P\right)_{T}$ from experimental results at different pressures, we first consider the enthalpies given in Table II. We used a five point algorithm for numerical derivatives (see Appendix B) to compute $c_{P}=(\partial h / \partial T)_{P}$ for each $T$ and $P$ of Table II. For the range of pressures considered, $c_{P}$ is a linear function of $P$ for each $T$. To reduce the numerical error in $\left(\partial c_{P} / \partial P\right)_{T}$, we made linear fits to $c_{P}(P)$ (see Appendix B) for each $T$. The values of $c_{P}$ at atmospheric pressure in Table I are included in these fits. The result is shown in Table III. The values of $\left(\partial c_{P} / \partial P\right)_{T}$ are negative, independent of the pressure. They are displayed in the second column of Table IV, which includes interpolated values for intermediate temperatures.

To compute $\left(\partial c_{P} / \partial P\right)_{T}$ at atmospheric pressure using Eq. (5), the derivative on the right-hand side with respect to $T$ at constant $P$ may be evaluated from the data of Table I using the same five points algorithm. The numerical derivative on the right-hand side of Eq. (5), with $c_{V}$ given by Eq. (3), was taken after the expression in brackets was evaluated. The result appears in the third column of Table IV.

Because the experimental data in Table I satisfy Mayer's relation for $c_{V}$, we can also evaluate $\left(\partial c_{P} / \partial P\right)_{T}$ using Eq. (6). In this case we have to obtain $\alpha^{\prime}$ numerically from $\alpha$ given in Table I. The result for $\left(\partial c_{P} / \partial P\right)_{T}$, using the five point algorithm, is the fourth column of Table IV. We take the latter result as the standard one because less numerical work is required (besides the empirical errors, only the numerical error of $\alpha^{\prime}$ is present.) The maximum relative deviation of the results for Eq. (5) relative to Eq. (6) is $0.6 \%$. However, the maximal deviation between the second and the fourth columns of Table IV is $16 \%$ and occurs at $T=0{ }^{\circ} \mathrm{C}$. These deviations are due to the inaccuracy in the derivatives of $h$ with respect to the temperature (the error is bigger at the end points), in the linear fits to $c_{P}$, and in the linear interpolations of $\left(\partial c_{P} / \partial P\right)_{T}$ for intermediate temperatures. A further reason for relying more on the last column of Table

Table III. Fits to $c_{P}(P)$ obtained from $c_{P}=(\partial h / \partial T)_{P}$ using the data given in Table II and the values of $c_{P}$ at atmospheric pressure from Table I.

\begin{tabular}{rc}
\hline \hline$T$ & $c_{P}$ \\
${ }^{\circ} \mathrm{C}$ & $\mathrm{J} \mathrm{kg}^{-1} \mathrm{~K}^{-1}$ \\
\hline 0 & $4217.2-4.1714 \times 10^{-6} P$ \\
20 & $4183.4-2.7913 \times 10^{-6} P$ \\
40 & $4177.1-2.3982 \times 10^{-6} P$ \\
60 & $4182.7-2.1642 \times 10^{-6} P$ \\
80 & $4196.6-2.0930 \times 10^{-6} P$ \\
100 & $4218.0-2.3722 \times 10^{-6} P$ \\
\hline
\end{tabular}

IV than on the other columns is the fact that the enthalpy and its variations are more difficult to measure than the volume and the expansion coefficient.

\section{CONCLUSIONS AND PROBLEMS}

We have used experimental data for liquid water to obtain $\left(\partial c_{P} / \partial P\right)_{T}$ using the first and second laws and compared the indirect results to direct measurements done at different pressures. We argued that Eq. (6) arising from the second law requires less information and gives more accurate numerical results. The two thermodynamic predictions for $\left(\partial c_{P} / \partial P\right)_{T}$ agree very well with each other and reasonably well with the values extracted from experiments at different pressures.

We expect thermodynamic relations to be universal. Because water shows anomalous behavior, ${ }^{17}$ testing thermodynamic relations using data on water provides a good test of thermodynamics. Similar data sets for other substances may also be used to check their thermodynamic consistency.

The questions studied here may help students better understand the formalism of thermodynamics. Students often become lost in the mathematical formalism and might not fully appreciate the physical content of the various thermodynamic relations. In contrast to engineering students, physics majors do not make much use of thermodynamic tables so that the connection of thermodynamics to experiment is limited. We think that it is educational to use empirical data and simple numerical methods to analyze it. To this end, we propose three problems for students.

Table IV. Comparison of values of $\left(\partial c_{P} / \partial P\right)_{T} \times 10^{6}$ (in $\mathrm{J} \mathrm{kg}^{-1} \mathrm{~K}^{-1} \mathrm{~Pa}^{-1}$ ) for water at $101.3 \mathrm{kPa}$. The second column was obtained from the slopes of the equations in Table III. The third column is obtained using Eq. (5) (first law) taking numerical derivatives of data in Table I. The last column is obtained from Eq. (6) (second law) using the same table. In the second column, linear interpolation was used for the temperatures not given in Table III. The most accurate results are in the last column.

\begin{tabular}{rccc}
\hline \hline $\begin{array}{c}T \\
{ }^{\circ} \mathrm{C}\end{array}$ & $\begin{array}{c}\text { From } \\
\text { Table III }\end{array}$ & $\begin{array}{c}\text { From } \\
\text { Eq. (5) }\end{array}$ & $\begin{array}{c}\text { From } \\
\text { Eq. (6) }\end{array}$ \\
\hline 0 & -4.171 & -4.935 & -4.942 \\
10 & -3.481 & -3.809 & -3.808 \\
20 & -2.791 & -3.127 & -3.118 \\
30 & -2.595 & -2.715 & -2.713 \\
40 & -2.398 & -2.462 & -2.463 \\
50 & -2.281 & -2.307 & -2.308 \\
60 & -2.164 & -2.219 & -2.220 \\
70 & -2.129 & -2.185 & -2.184 \\
80 & -2.093 & -2.190 & -2.187 \\
90 & -2.233 & -2.233 & -2.228 \\
100 & -2.372 & -2.306 & -2.319 \\
\hline \hline
\end{tabular}


Table V. Experimental data of $\mathrm{V}$ and $\alpha$ for water at $101.3 \mathrm{kPa}$ for temperatures close to $0{ }^{\circ} \mathrm{C}$ (to be used in Problem 1) (Ref. 18).

\begin{tabular}{cc}
\hline \hline$T$ & $\begin{array}{c}\mathrm{v} \times 10^{3} \\
\mathrm{~m}^{3} \mathrm{~kg}^{-1}\end{array}$ \\
${ }^{\circ} \mathrm{C}$ & 1.000160 \\
0 & 1.000100 \\
1 & 1.000060 \\
2 & 1.000036 \\
3 & 1.000028 \\
5 & 1.000036 \\
\hline
\end{tabular}

(1) Water has a density maximum at $4{ }^{\circ} \mathrm{C}$ (Table V). Use the five points algorithm given in Appendix B to evaluate $\alpha$ and $\alpha^{\prime}$ for the temperatures indicated in the table. Evaluate the right-hand side of Eq. (6) at $0{ }^{\circ} \mathrm{C}$ and compare the result with the corresponding ones in Table IV.

(2) From the data of Table II obtain $(\partial h / \partial P)_{T}$ by fitting values at the same temperature and different pressures. Then evaluate the same quantity at atmospheric pressure from Eq. (9) (first law) and from Eq. (13) (second law) using the data of Table I.

(3) Insert the compatibility condition, Eq. (13), in Eq. (11). Use a numerical integration algorithm, ${ }^{19}$ to integrate Eq. (11) and construct a table for the entropy $s(T, P)$ from the data in Table II. Take $s(T=273.15 \mathrm{~K}, P$ $=5.00 \mathrm{MPa})=0.0001 \mathrm{~kJ} \mathrm{~kg}^{-1} \mathrm{~K}^{-1} .{ }^{16}$

\section{APPENDIX A: PLANCK'S RESULT}

Based on the first law only, Planck established the following relation between thermal coefficients and specific heat: ${ }^{14}$

$$
\begin{aligned}
& \left(c_{P}-c_{V}\right) \frac{\partial^{2} T}{\partial P \partial \mathrm{v}}+\left(\frac{\partial c_{P}}{\partial P}\right)_{V}\left(\frac{\partial T}{\partial \mathrm{v}}\right)_{P}-\left(\frac{\partial c_{V}}{\partial \mathrm{v}}\right)_{P}\left(\frac{\partial T}{\partial P}\right)_{V} \\
& =1 \quad \text { (Planck's result). }
\end{aligned}
$$

Here we derive this result and show that it is equivalent to Eq. (5).

Taking the specific internal energy $u$ as a function of the specific volume $\mathrm{V}$ and pressure $P$, we may write

$$
\left(\frac{\partial u}{\partial P}\right)_{\mathrm{v}}=c_{V}\left(\frac{\partial T}{\partial P}\right)_{\mathrm{v}} \text {. }
$$

From $d u=\delta q-P d \mathrm{v}$, we obtain

$$
\left(\frac{\partial u}{\partial \mathrm{v}}\right)_{P}=c_{P}\left(\frac{\partial T}{\partial \mathrm{v}}\right)_{P}-P
$$

Taking derivatives of Eqs. (15) and (16) and applying Schwartz's theorem, we obtain

$$
\left\{\frac{\partial}{\partial \mathrm{V}}\left[c_{V}\left(\frac{\partial T}{\partial P}\right){ }_{\mathrm{V}}\right]\right\}_{P}=\left\{\frac{\partial}{\partial P}\left[c_{P}\left(\frac{\partial T}{\partial \mathrm{V}}\right)_{P}-P\right]\right\}_{\mathrm{V}},
$$

or

$\left(\frac{\partial c_{V}}{\partial \mathrm{V}}\right)_{P}\left(\frac{\partial T}{\partial P}\right)_{\mathrm{V}}+c_{V} \frac{\partial^{2} T}{\partial \mathrm{v} \partial P}=\left(\frac{\partial c_{P}}{\partial P}\right)_{\mathrm{V}}\left(\frac{\partial T}{\partial \mathrm{V}}\right)_{P}+c_{P} \frac{\partial^{2} T}{\partial P \partial \mathrm{V}}-1$.
By applying Schwartz's theorem to $T=T(P, \mathrm{~V})$, we obtain Planck's result (14).

Now we use $T$ and $P$ as independent variables to verify that Eq. (18) is equivalent to Eq. (5). From the definitions of $\alpha$ and $\kappa_{T}$ and Eq. (1), we have

$$
\left(\frac{\partial c_{V}}{\partial \mathrm{V}}\right)_{P}\left(\frac{\partial T}{\partial P}\right)_{\mathrm{V}}=\left(\frac{\partial c_{V}}{\partial T}\right)_{P}\left(\frac{\partial T}{\partial \mathrm{V}}\right)_{P}\left(\frac{\partial T}{\partial P}\right)_{\mathrm{V}}=\frac{c_{\mathrm{v}}^{\prime} \kappa_{T}}{\alpha^{2} \mathrm{~V}} .
$$

Moreover,

$$
\frac{\partial^{2} T}{\partial \mathrm{V} \partial P}=\left[\frac{\partial}{\partial \mathrm{V}}\left(\frac{\kappa_{T}}{\alpha}\right)\right]_{P}=\left[\frac{\partial}{\partial T}\left(\frac{\kappa_{T}}{\alpha}\right)\right]_{P}\left(\frac{\partial T}{\partial \mathrm{V}}\right)_{P}=\frac{1}{\alpha \mathrm{V}}\left(\frac{\kappa_{T}}{\alpha}\right)^{\prime},
$$

and the left-hand side of Eq. (18) becomes

$$
c_{V}^{\prime} \frac{\kappa_{T}}{\alpha^{2} \mathrm{v}}+\frac{c_{V}}{\alpha \mathrm{v}}\left(\frac{\kappa_{T}}{\alpha}\right)^{\prime}=\frac{1}{\alpha \mathrm{v}}\left(\frac{c_{V} \kappa_{T}}{\alpha}\right)^{\prime}
$$

On the other hand, we have

$$
\left(\frac{\partial c_{P}}{\partial P}\right)_{\mathrm{v}}\left(\frac{\partial T}{\partial \mathrm{v}}\right)_{P}=\left[\left(\frac{\partial c_{P}}{\partial P}\right)_{T}+c_{P}^{\prime} \frac{\kappa_{T}}{\alpha}\right] \frac{1}{\alpha \mathrm{V}}
$$

and the right-hand side of Eq. (18) is

$$
\begin{gathered}
\frac{1}{\alpha \mathrm{v}}\left[\left(\frac{\partial c_{P}}{\partial P}\right)_{T}+c_{P}^{\prime} \frac{\kappa_{T}}{\alpha}\right]+\frac{c_{P}}{\alpha \mathrm{v}}\left(\frac{\kappa_{T}}{\alpha}\right)^{\prime}-1 \\
=\frac{1}{\alpha \mathrm{v}}\left(\frac{c_{P} \kappa_{T}}{\alpha}\right)^{\prime}+\frac{1}{\alpha \mathrm{v}}\left(\frac{\partial c_{P}}{\partial P}\right)_{T}-1 .
\end{gathered}
$$

If we equate Eqs. (21) and (23) and reorder terms, we obtain Eq. (5).

\section{APPENDIX B: NUMERICAL METHODS}

Given $N$ data points for the quantity $y(x)$, the derivative $d y / d x$ can be obtained numerically by using the five point algorithm: ${ }^{20}$

$$
\begin{aligned}
\left(\frac{d y}{d x}\right)_{1}= & \frac{1}{24 \Delta x}\left(-50 y_{1}+96 y_{2}-72 y_{3}+32 y_{4}-6 y_{5}\right), \\
\left(\frac{d y}{d x}\right)_{2}= & \frac{1}{24 \Delta x}\left(-6 y_{1}-20 y_{2}+36 y_{3}-12 y_{4}+2 y_{5}\right), \\
\left(\frac{d y}{d x}\right)_{i}= & \frac{1}{24 \Delta x}\left(2 y_{i-2}-16 y_{i-1}+16 y_{i+1}-2 y_{i+2}\right), \\
\left(\frac{d y}{d x}\right)_{N-1}=\frac{1}{24 \Delta x}\left(-2 y_{N-4}+12 y_{N-3}-36 y_{N-2}\right. & \left.\quad+20 y_{N-1}+6 y_{N}\right) \\
\left(\frac{d y}{d x}\right)_{N}= & \frac{1}{24 \Delta x}\left(6 y_{N-4}-32 y_{N-3}+72 y_{N-2}\right. \\
& \left.-96 y_{N-1}+50 y_{N}\right) .
\end{aligned}
$$

The algorithm also applies to partial derivatives. This algorithm was implemented using Excel.

We used the analytical equation of state of Thomsen and Hartka $^{21}$ to estimate the error for $\alpha^{\prime}$ at $T=0^{\circ} \mathrm{C}$ and $P$ $=101.3 \mathrm{kPa}$. For a temperature step of $10^{\circ} \mathrm{C}$, the result 
$\alpha^{\prime}\left(T=0{ }^{\circ} \mathrm{C}\right)=16.0001 \times 10^{-6} \mathrm{~K}^{-2}$ obtained with the five point algorithm is accurate to within $2 \times 10^{-10} \mathrm{~K}^{-2}$.

Excel was also used to fit data for $c_{P}(P)$ to a straight line (linear regression). The use of spreadsheets is appropriate for the type of analysis presented in this paper and requires only modest knowledge of computer programming.

${ }^{1}$ M. W. Zemansky and R. H. Dittman, Heat and Thermodynamics (McGraw-Hill, New York, 1997), 7th ed., Chap. 9. This book was reviewed in Am. J. Phys. 66, 164-166 (1998).

${ }^{2}$ H. B. Carroll, M. Eisner, and R. M. Henson, "Rubber band experiment in thermodynamics,” Am. J. Phys. 31, 808-808 (1963).

${ }^{3}$ B. Bergthorsson, "Temperature, transitivity, and the zeroth law," Am. J. Phys. 45, 270-271 (1977).

${ }^{4}$ F. W. Sears, and G. Salinger, Thermodynamics, Kinetic Theory, and Statistical Thermodynamics (Addison-Wesley, Reading, MA, 1975), pp. 101102 .

${ }^{5} \mathrm{C}$. Truesdell, The Tragicomical History of Thermodynamics 1822-1854 (Springer, New York, 1980), p. 189. Ferdinand Reech (1805-1884) was a French naval engineer, whose name is associated with Eq. (3) in the French literature.

${ }^{6}$ The subscript $s$ simply means that the process is adiabatic and reversible; we do not need the notion of entropy here. See Ref. 1, p. 239.

${ }^{7} \mathrm{~K}$. Weltner, "Measurement of specific heat capacity of air," Am. J. Phys. 61, 661-662 (1993).

${ }^{8} \mathrm{D}$. J. Cronin, "The temperature variation of gamma for various gases: a student experiment," Am. J. Phys. 32, 700-704 (1964).

${ }^{9}$ S. Velasco, F. L. Román, and J. Faro, "A simple experiment for measuring the adiabatic coefficient of air," Am. J. Phys. 66, 642-643 (1998).

${ }^{10}$ Reference 1, p. 270.

${ }^{11}$ G. Weinreich, Fundamental Thermodynamics (Addison-Wesley, Reading, MA, 1968), notes that "The fact that such a relation is mathematically an almost trivial identity should not blind us to the fact that there is nothing trivial physically about it or about other thermodynamic identities such as the Maxwell relations. The experiments which are used to obtain heat capacities are completely different from those that give the expansion coefficients. That the resulting numerical values consistently satisfy that relation must, accordingly, be viewed as the working of a deep physical law."

${ }^{12}$ A. J. Mallinckrodt and H. S. Leff, "All about work," Am. J. Phys. 60, 356-365 (1992).

${ }^{13}$ Furthermore, for water at normal pressure and in the temperature range $0-8{ }^{\circ} \mathrm{C}$, $\mathrm{v}$ and $P$ cannot be independent variables because there are two values of $T$ corresponding to the same $\mathrm{v}$.

${ }^{14}$ M. Planck, Treatise on Thermodynamics (Dover, Mineola, 1990), p. 59, who wrote "This equation contains only quantities which may be experimentally determined, and therefore furnishes a means for testing the first law of thermodynamics by observations on any homogeneous substance."

${ }^{15}$ Reference 1, p. 242.

${ }^{16}$ J. H. Keenan et al., Steam Tables. Thermodynamic Properties of Water Including Vapor, Liquid, and Solid Phases (Wiley, New York, 1969), pp. 104-105. These data are transcribed in text books, such as A. Shavit and C. Gutfinger, Thermodynamics. From Concepts to Applications (PrenticeHall, Europe, 1995), pp. 452-453. However, one must be aware of transcription errors. We found two in the latter book: one for $\mathrm{v}(T$ $\left.=100{ }^{\circ} \mathrm{C}, P=5.00 \mathrm{MPa}\right)$ and the other for $\mathrm{v}\left(T=20^{\circ} \mathrm{C}, P=200 \mathrm{MPa}\right)$.

${ }^{17} \mathrm{H}$. Tanaka, "Simple physical explanation of the unusual thermodynamic behavior of liquid water," Phys. Rev. Lett. 80, 5750-5753 (1998).

${ }^{18}$ E. P. Gyftopoulos and G. P. Beretta, Thermodynamics: Foundations and Applications (MacMillan, New York, 1991), pp. 642-645.

${ }^{19}$ F. S. Acton, Numerical Methods that Work (The Mathematical Association of America, Washington, DC, 1990), Chap. 10.

${ }^{20}$ Handbook of Mathematical Functions with Formulas, Graphs, and Mathematical Tables, edited by M. Abramowitz and I. A. Stegun (Dover, New York, 1972), p. 914.

${ }^{21}$ J. S. Thomsen and T. J. Hartka, "Strange Carnot cycles; Thermodynamics of a system with a density extremum," Am. J. Phys. 30, 26-33 (1962).

\section{IS QUANTUM THEORY ROTTEN?}

I also told Wheeler that from the time that Bell began to study the quantum theory, he had conceptual problems with it, and that I had asked Bell if, at that time, he thought that the theory might simply be wrong - to which Bell had answered, "I hesitated to think it might be wrong, but I knew that it was rotten."

Jeremy Bernstein, “John Wheeler: Retarded Learner,' in Quantum Profiles (Princeton University Press, Princeton, 1991), p. 137. 